\title{
BLOCK COPOLYMERIC MICELlES: BASIC CONCEPT AND Preparation TeChNiQues
}

\author{
Sunita Dahiya* \\ Department of Pharmaceutical Sciences, School of Pharmacy, Medical Sciences Campus, \\ University of Puerto Rico, San Juan, PR 00936-5067, USA \\ *E-mail: sunita.dahiya@upr.edu \\ Tel.: +1 7877582525 ext. 5413.
}

Received: Nov 14, 2019 / Revised: Dec 19, 2019 / Accepted: Dec 20, 2019

\begin{abstract}
Block copolymeric micelles, the nanoscopic core-shell structures formed by amphiphilic block copolymers, are one of the most well-suited drug delivery carriers among polymeric nanoparticles due to their inherent and modifiable properties. These nanostructures are capable of entrapping wide range of drugs and find limitless applications in the area of drug delivery. The purpose of this article is to briefly highlight basic concept and preparation techniques of polymeric micelles prepared by employing block copolymers.
\end{abstract}

Key words: Polymeric micelle, Block copolymer, Solvent evaporation, Flash nanoprecipitation.

\section{INTRODUCTION}

Since past few decades, the practice of drug delivery has changed dramatically and even greater changes are anticipated in the near and far future. Breakthroughs in medicine and drug delivery would not have been possible without research contributions of various fields including biomedical engineering, polymer and material chemistry, biophysics, biotechnology and molecular pharmacology. These multidisciplinary areas enabled substantially to the understanding of the physiological barriers to efficient drug delivery, such as transport in the circulatory system and drug movement through cells and tissues; mechanisms of drug release and in vivo performance, and development of novel patient-friendly drug delivery devices. Our research group has reported several findings to address solubility issues associated with formulation and delivery aspects of immediate and extended release formulations for poorly soluble drugs (Dahiya, 2006; Dahiya and Pathak, 2006a; 2006b; Dahiya and Pathak, 2007; Pathak et al 2008; Jain et al 2010; Dahiya and Tayde, 2013; Dahiya et al
2015; Dahiya et al 2019), and freely water soluble drugs for extended delivery (Dahiya et al 2008; Dahiya and Tyagi, 2008; Dahiya et al 2011; Dahiya and Gupta, 2011; Dahiya and Onker, 2014) using solid dispersion and complexation approaches. Besides, enormous research findings report nanotechnological advancements to address drug delivery challenges associated with poor solubility and poor bioavailability. Among several nanotechnology-based techniques, polymeric micelles have drawn much attention for delivery of poorly water soluble drugs (Verma and Hassan, 2013) and have been studied extensively for drug delivery applications including Paclitaxel, Indomethacin, Amphotericin B, Adriamycin, and Dihydrotestosterone for injectable formulations of polymeric micelles (Mourya et al 1998; Yu et al 1998; Allen et al 2000; Bae et al 2005).

Polymeric micelles, the nanoscopic core-shell structures formed by amphiphilic block copolymers, are one of the most well-suited drug delivery carriers among polymeric nanoparticles due to their inherent and modifiable properties. 
A copolymer is a polymer derived from more than one species of monomer whereas a block copolymer is a copolymer formed when the two monomers cluster together and form 'blocks' of repeating units. The highly controlled polymerization techniques such as atom transfer radical polymerization (ATRP) (Siegwart, 2012) and reversible addition fragmentation chain transfer (RAFT) (Gregory and Stenzel, 2012) enabled syntheses of block copolymers structures to fit biomedical applications as well as formation of diversified self-assembled morphologies such as micelles, cylindrical micelles, and polymersomes. Various nanostructured carriers such as liposomes, polymer-drug conjugates, nanoparticles, and polymeric micelles have been explored for their drug delivery applications including targeted drug delivery to tumors (Kataoka et al 2001; Langer, 2007; Davis et al 2008). These nanostructures offer great applications starting from solubility improvement of drugs to the tailoring of pharmacokinetics to attain reduced toxicities of the drugs. The successful clinical translation of several of these nanomedicines further supports the strategy as an efficient therapeutic technique. Polymeric micelles have demonstrated unique advantages for incorporating a wide range of bioactive molecules and overcoming any biological barriers. The special feature of micelles is the core-shell structure that is highly water soluble while still maintaining a hydrophobic core suitable for hydrophobic drugs (Lu and Park, 2013; Kowalczuk et al 2014).

\section{Micelles and polymeric micelles}

The differences between the solubility of the hydrophilic and hydrophobic blocks of an amphiphilic copolymer in an aqueous solution drive the formation of distinctive and unique polymeric micelles with a core-shell architecture as depicted in Figure 1a, $\mathbf{1 b}$. Amphiphilic block or graft copolymers also behave in the same manner as that of conventional amphiphiles or surfactant molecules and these polymers form polymeric micelles in aqueous solution above CMC. Polymeric micelles are nano-sized colloidal carrier system which comprise of polymer chains and are formed from amphiphilic block copolymers which comprises of both hydrophilic and hydrophobic monomer units, such as polyethylene oxide (PEO) and polypropylene oxide (PPO), respectively. These are spontaneously formed by self-assembly in a liquid, generally as a result of hydrophobic or ion pair interactions between polymer segments. Polymeric micelles usually have a core-shell structure, in which core contains either the hydrophobic part or the ionic part of the nanoparticles, and can possess either small or bigger molecules such as therapeutic active drugs, while the shell provides interactions with the solvent and construct the nanoparticles (Mourya et al 2010).

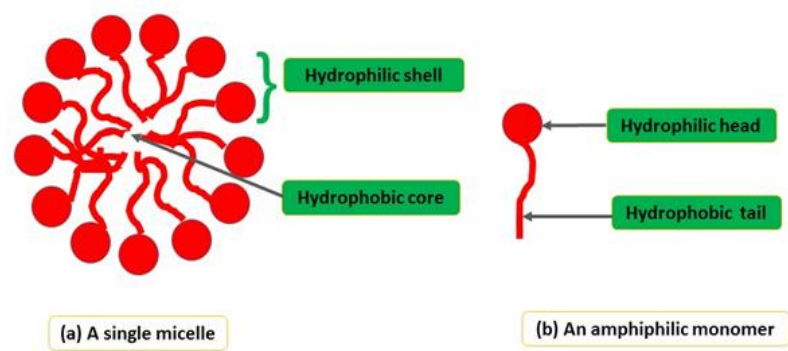

Fig. 1. Illustration of (a) a single micelle with core-shell structure, (b) an amphiphilic monomer

Spontaneous formation of micelles takes place when surfactant molecules are dissolved in water at concentrations above the critical micelle concentration (CMC). CMC is defined as the concentration of surfactants at and above which micelles form and almost all additional surfactants added to the system go to form micelles. The spontaneous formation of micelles from amphiphilic copolymers in aqueous media, when the CMC is achieved, is shown in Figure 2.

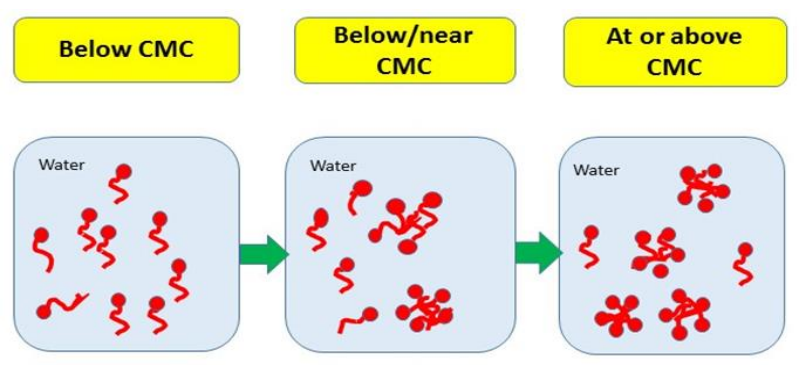

Fig. 2. Spontaneous micelle formation in water

Unlike the micelles of conventional surfactant monomers, polymeric micelles do possess a covalent linkage in individual surfactant molecules within the hydrophobic core. This linkage prevents dynamic exchange of monomers between free solution and the micellar pseudo-phase. This confers rigidity and stability to the polymeric micelles. Other advantages of polymeric micelles over conventional surfactant micelles are depicted in Figure 3. 


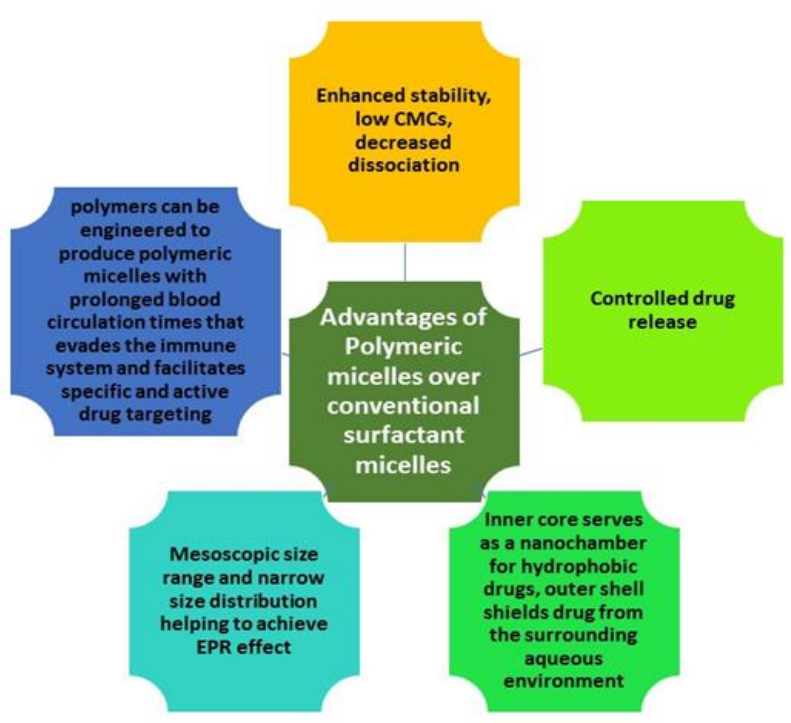

Fig. 3. Advantages of polymeric micelles over surfactant micelles

The aggregation number of polymeric micelles can be several hundreds and the diameter ranges from 10 to $100 \mathrm{~nm}$. Factors controlling the size of the polymeric micelles include molecular weight of the amphiphilic block copolymer, aggregation number of the amphiphiles, relative proportion of hydrophilic and hydrophobic chains, and the preparation process (Jones and Leroux, 1999).

\section{Selection of block copolymers}

A range of commercially available block copolymers may be employed for the design of amphiphilic structures. The fabrication of polymeric micelles mainly involves the use of amphiphilic diblock copolymers, although graft and triblock copolymers can also be used. These three copolymer types possess exclusive benefits that can be utilized for the delivery of drugs, such as the prolongation of drug circulation time, control of the drug-release profile, or the ability to add targeting ligands. In addition, block copolymers can be further complemented by other amphiphilic polymers such as star polymers, miktoarm star polymers, and multiblock copolymers, to enable the formation of compartmentalized micelles. Irrespective of the architecture chosen, the basic consideration for the polymer selection should be the compatibility between the drug and the polymers (Kowalczuk et al 2014) because the polymer-drug interaction plays an important role in the drug-loading capacity of a carrier and the stability of the drug in the matrix which in turn affects the shelf-life of the carrier. Another important parameter is the miscibility of a drug with the polymeric matrix. This can be described by the Flory-Huggins theory which contains both entropy and enthalpy components, expressed by the Flory-Huggins interaction parameter $\chi$ that describes the interaction between the polymer and the drug. In other words, the Flory-Huggins parameter $\chi$ is a measure of compatibility between polymer and drug. However, many drugs have a strong tendency to crystallize where the presence of the homogenous mixture is determined by the miscibility curve of its phase diagram which is based on fast equilibrium. It does not usually happen in case when the polymers with high glass transition temperature $\left(\mathrm{T}_{\mathrm{g}}\right)$ values traps the drug in the matrix resulting in a kinetically stable system.

In this scenario, the most suitable way to start with is "like dissolves like" based on the Scatchard-Hildebrand solubility parameter of the drug and polymer, indicating that the polymers that are chemically similar to the drug should enable the highest loading capacity. Polymeric micelles have been specifically explored in order to facilitate the delivery of hydrophobic drugs. Suitable amphiphilic block copolymers are obtainable via controlled synthesis by varying the block ratio, the total molecular weight, and the chemical structure. By adjusting the structure of the amphiphilic copolymers, the size and morphology of the resulting polymeric micelles can be easily controlled. Various classes of block copolymer commonly employed for the fabrication of polymeric micelles are summarized in Table 1 (Croy and Kwon, 2006).

\section{Preparation techniques of drug-loaded polymeric micelle}

Drugs that have a low drug loading efficiency are best delivered by conjugating them to the block copolymer directly, instead of relying on physical attraction alone. The direct mixing of the hydrophobic drug and the micelle in water is suitable for some selected systems, however, it is rarely capable of dissolving both the drug and polymer. Therefore, other drug loading techniques must be used to ensure solubilization of both the drug and the polymer. A common solvent is capable of fully dissolving both the drug and the block copolymer into the monomeric state. A clear solution serves as an initial indication that the polymer has dissolved, however, it is advisable to test for the absence of micelles or other aggregates using light scattering techniques to ensure full solvation. 
Schematics of drug-loading techniques are described in Figure 4. The brief description of various preparation techniques with their example methods are discussed.

Table 1. Commonly employed block copolymers for polymeric micelles

\begin{tabular}{|c|c|c|}
\hline Polymer & Core forming block & Features \\
\hline Poly(ester)s & $\begin{array}{c}\text { poly(D,L-lactic acid-co-glycolic acid) } \\
\text { poly(D,L-lactide) } \\
\text { poly( } \varepsilon \text {-caprolactone })\end{array}$ & $\begin{array}{l}\text { Susceptible to hydrolysis, resulting } \\
\text { in degradation to non-toxic species } \\
\text { and leading to the expectation for } \\
\text { safe systemic administration } \\
\\
\text { The poly(ester)s are not as } \\
\text { amenable to chemical } \\
\text { modifications as } \\
\text { poly(L-amino acid) }\end{array}$ \\
\hline poly(L-amino acid)s & $\begin{array}{c}\text { poly(L-aspartate }) \\
\text { poly(2-hydroxyalkyl-L-aspartamide }) \\
\text { poly( } \beta \text {-benzyl-L-aspartate }) \\
\text { poly }(\beta \text {-benzyl-L-glutamate })\end{array}$ & $\begin{array}{l}\text { Amino acids may be biodegradable } \\
\text { into naturally occurring substances } \\
\text { in the body by hydrolysis or } \\
\text { enzymatic degradation } \\
\text { Free amine or carboxylic acid } \\
\text { functional groups of the amino } \\
\text { acids can be chemically tailored to } \\
\text { improve compatibility with the } \\
\text { intended solubilizate or to improve } \\
\text { the stability of the micelle }\end{array}$ \\
\hline poloxamers & $\begin{array}{l}\text { P85, P105 } \\
\text { L61 } \\
\text { F127, F98 } \\
\text { F87, F68 }\end{array}$ & $\begin{array}{l}\text { Well-soluble in aqueous systems } \\
\text { and form micelles spontaneously } \\
\text { upon direct addition to water, } \\
\text { allowing for simple preparation } \\
\\
\text { Do not biodegrade upon } \\
\text { administration }\end{array}$ \\
\hline
\end{tabular}

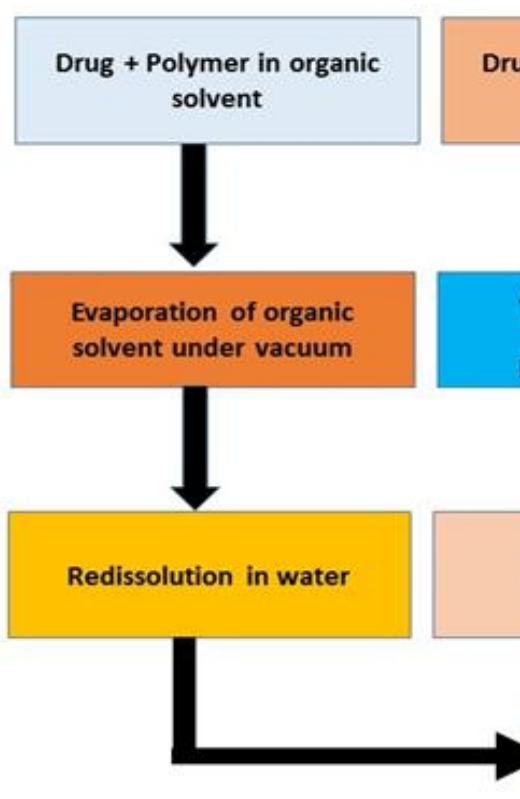

(a)
Drug + Polymer in organic solvent

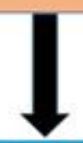

Addition of organic phase into water phase or vice versa

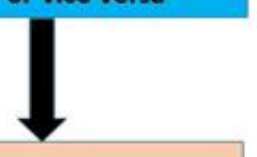

Evaporation of organic solvent

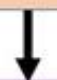

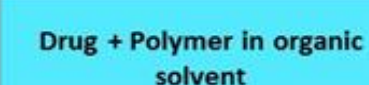
solvent

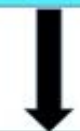

Slow addition of water to organic solvent containing drug and polymer

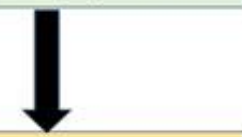

Removal of organic solvent by dialysis

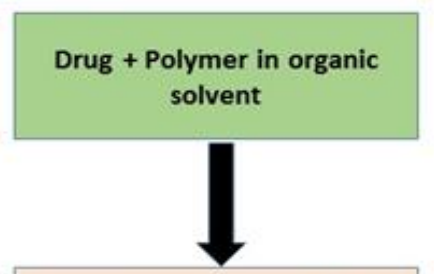

Fast mixing of organic Phase with water using confined impinging jet mixer

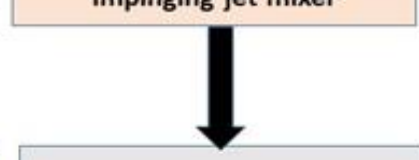

Removal of organic solvent by dialysis

\section{Drug-loaded polymeric micelles}

(b)

(c)

(d)

Fig. 4. Schematic representation of polymeric micelles' preparation techniques (a) solvent evaporation, (b) co-solvent evaporation, (c) Illustration of (a) a single micelle with core-shell structure, (b) an amphiphilic monomer) dialysis, (d) flash nanoprecipitation 


\section{Solvent evaporation technique}

In the solvent evaporation technique, both polymer and drug are dissolved in an organic solvent with a low boiling point, followed by evaporation and subsequent dehydration (Pérez et al 2002). The chosen organic solvent is selective toward one block, which results in the formation of micelles in non-aqueous solutions. The product quality is influenced by the type of solvent, the concentration of polymer and drug, and the rate of evaporation. The limitation of this approach lies in the limited choice of solvents, and there is no guarantee the resulting particles will be well-defined core-shell particles that can be easily re-dissolved in water. For example, methanol or any other low-boiling solvent that can dissolve both components may be employed for this technique.

\section{Co-solvent evaporation technique}

Co-solvent evaporation proceeds by adding water directly to the organic solvent to achieve the self-assembly of the micelle and encapsulation of the drug. The outcome is controlled by the type of solvent, the ratio between organic solvent and water, the concentration of water and drug, rate of solvent evaporation, and the order and rate of mixing (Boury et al 1995). Although this approach is limited by the choice of solvent, it usually results in higher drug encapsulation efficiencies. For example, acetone, tetra hydro furan (THF) or acetonitrile can be used as organic solvent. Also, the addition of water is dropwise to the organic solvent or vice versa and mixed for at least four hours before evaporating the organic solvent.

\section{Dialysis}

Dialysis is one of the most versatile and most common technique used for drug encapsulation since it allows the use of high-boiling solvents such as dimethylsulfoxide (DMSO) which is removed by dialysis and replaced with water. Dialysis approach is applicable to many solvent systems and the drug loading efficiency is usually lower than the co-solvent evaporation. Further, the technique can be slow which can aid the formation of thermodynamically stable morphologies. The final dialysis step is done to remove solvent and collect the free drug. This step is often crucial to obtain a product free of

\section{REFERENCES}

Allen C, Han J, Yu Y, Maysinger D, Eisenberg A. Polycaprolactone-b-poly(ethylene oxide) copolymer micelles as a delivery vehicle for dihydrotestosterone. organic solvent while maintaining maximum drug loading. If extensive dialysis is performed, it can assist in the thorough purification of the product, but it also can cause the release of the already encapsulated drug, giving rise to low drug encapsulation efficiencies. The example method for dialysis is given as follows. For example, organic solvent like dimethyl formamide (DMF) can be used in this method. The rate of addition of water can be controlled using a syringe pump. Finally, the solution is dialyzed against water using a tubular cellulose membrane.

\section{Flash nanoprecipitation}

Flash nanoprecipitation is a relatively new technique that offers a more rapid solution than other time-consuming methods as it involves fast mixing and precipitation into a non-solvent for the drug and one polymer block resulting in a kinetically trapped structure. Although, this resulting structures do not have well-defined internal phase boundaries like thermodynamically stable structures, the approach provides an alternative to achieve a fast throughput (York et al 2012). For example, THF can be used as organic solvent and the solution is mixed with water using a confined impinging jet mixer. The exit stream is introduced into water:THF $(9: 1 \% v / v)$ followed by dialysis against water using a tubular cellulose membrane.

\section{CONCLUSIONS}

Block copolymeric micelles are being explored for the drug delivery applications, specifically for the enhancement of solubility of the hydrophobic drugs. The efficiency of drug loading in these micelles depends on compatibility between the drug and the block copolymer employed. The drugs that have a low drug loading efficiency are best delivered by conjugating them to the block copolymer directly, instead of relying on physical attraction alone. Polymeric micelles can be prepared by various techniques such as solvent and cosolvent evaporation, flash nanoprecipitation and dialysis. The polymeric micelles are versatile nanosystems that demonstrate limitless avenues for modification enabling delivery of wide varieties of therapeutic agents.

J. Control. Release 2000;63(3):275-86. [DOI: 10.1016/s01 68-3659(99)00200-x]

Bae Y, Jang WD, Nishiyama N, Fukushimaa S, Kataoka K. 
Multifunctional polymeric micelles with folate-mediated cancer cell targeting and pH-triggered drug releasing properties for active intracellular drug delivery. Mol. BioSyst. 2005;1(3):242-50. [DOI: 10.1039/b500266d]

Boury F, Ivanova, T, Panaieotov I, Proust JE, Bois A, Richou J. Dilatational properties of adsorbed poly(D,L-lactide) and bovine serum albumin monolayers at the dichloromethane/water interface. Langmuir 1995;11(5): 1636-1644. [DOI: 10.1021/la0000 5a036]

Croy SR, Kwon GS. Polymeric micelles for drug delivery. Curr. Pharm. Design 2006;12:4669-4684. [DOI: 10.2174/ 1381 61206779026245]

Dahiya S, Asati S, Mallurwar V. Formulation and evaluation of granisetron hydrochloride orodispersible tablets. Bull. Pharm. Res. 2011;1(2):41-6.

Dahiya S, Gupta ON. Formulation and in vitro evaluation of metoprolol tartrate microspheres. Bull. Pharm. Res. 2011;1(1):31-9.

Dahiya S, Kaushik A, Pathak K. Formulation optimization of multicomponent aqueous co-ground mixtures of meloxicam for dissolution enhancement. Chem. Biol. Lett. 2019;6(1):1-7.

Dahiya S, Kaushik A, Pathak K. Improved pharmacokinetics of aceclofenac immediate release tablets incorporating its inclusion complex with hydroxypropyl- $\beta$-cyclodextrin. Sci. Pharm. 2015;83:501-10.

Dahiya S, Onker R. Influence of polymethacrylates and compritol on release profile of a highly water soluble drug metformin hydrochloride. Ars Pharm. 2015;56(1):24-31.

Dahiya S, Pathak K. Influence of amorphous cyclodextrin derivatives on aceclofenac release from directly compressible tablets. Pharmazie 2007;62(4):278-83.

Dahiya S, Pathak K. Physicochemical characterization and dissolution enhancement of aceclofenac-hydroxypropyl beta-cyclodextrin binary systems. PDA J. Pharm. Sci. Tech. 2006a;60(6):378-88.

Dahiya S, Pathak K. Dissolution enhancement of aceclofenac by $\beta$-cyclodextrin complexation. J. Pharm. Res. 2006b; 5(4):99-103.

Dahiya S, Tayde P. Binary and ternary solid systems of carvedilol with 2-hydroxypropyl- $\beta$-cyclodextrin and PVP K30. Bull. Pharm. Res. 2013;3(3):128-34.

Dahiya S, Tyagi L. Preparation and evaluation of oxytetracycline hydrochloride microbeads for delayed release. Pak. J. Pharm. Sci. 2008;21(2):103-8.

Dahiya S. Formulation and evaluation of release retardant matrix tablets of diclofenac sodium. FABAD J. Pharm. Sci. 2006;31(3):119-26.

Dahiya S. Fundamentals of solubility enhancement techniques: what do we need to consider? Bull. Pharm. Res. 2017;7(1):138. [DOI: 10.21276/bpr.2017.7.1.1]

Dahiya S. Studies on formulation development of a poorly water-soluble drug through solid dispersion technique. Thai J. Pharm. Sci. 2010;34(2):77-87.

Dahiya S, Pathak K, Sharma R. Development of extended release coevaporates and coprecipitates of promethazine $\mathrm{HCl}$ with acrylic polymers: formulation considerations. Chem. Pharm. Bull. (Tokyo) 2008;56(4):504-8.

Davis ME, Chen Z, Shin D. Nanoparticle therapeutics: an emerging treatment modality for cancer. Nat. Rev. Drug
Discov. 2008;7(9):771-82. [DOI: 10.1038/nrd2614]

Gregory A, Stenzel MH. Complex polymer architectures via RAFT polymerization: from fundamental process to extending the scope using click chemistry and nature's building blocks. Prog. Polym. Sci. 2012;37(1):38-105. [DOI: 10.1016/j.progpolymsci.2011.08.004]

Jones MC, Leroux JC. Polymeric micelles- a new generation of colloidal drug carriers. Eur. J. Pharm. Biopharm. 1999;48(2):101-11. [DOI: 10.1016/s0939-6411(99)0003 9-9]

Kataoka K, Harada A, Nagasaki Y. Block copolymer micelles for drug delivery: design, characterization and biological significance. Adv. Drug Deliv. Rev. 2001;47(1):113-31. [DOI: 10.1016/s0169-409x(00)00124-1]

Kim SY, Shin IG, Lee YM, Cho CS, Sung YK. Methoxy poly(ethylene glycol) and epsilon-caprolactone amphiphilic block copolymeric micelle containing indomethacin. II. Micelle formation and drug release behaviours. J. Control. Release 1998;51(1):13-22. [DOI: 10.1016/s0168-3659(97)00124-7]

Kowalczuk A, Trzcinska R, Trzebicka B, Müller AHE, Dworak A, Tsvetanov CB. Loading of polymer nanocarriers: factors, mechanisms and applications. Prog. Polym. Sci. 2014;39(1):43-86. [DOI: 10.1016/j.progpoly msci.2013.10.004]

Lu Y, Park K. Polymeric micelles and alternative nanonized delivery vehicles for poorly soluble drugs. Int. J. Pharm. 2013;453(1):198-214. [DOI: 10.1016/j.ijpharm.2012.08. 042]

Mourya VK, Inamdar N, Nawale RB, Kulthe SS. Polymeric micelles: general considerations and their applications. Ind. J. Pharm. Edu. Res. 2011;45(2):128-38.

Peer D, Karp JM, Hong S, Farokhzad OC, Margalit R, Langer R. Nanocarriers as an emerging platform for cancer therapy. Nat. Nanotechnol. 2007;2(12):751-60. [DOI: 10.1 038/nnano.2007.387]

Pérez C, Castellanos IJ, Costantino HR, Al-Azzam W, Griebenow K. Recent trends in stabilizing protein structure upon encapsulation and release from bioerodible polymers. J. Pharm. Pharmacol. 2002;54:30113. [DOI: 10.1211/0022357021778448]

Siegwart DJ, Oh JK, Matyjaszewski K. ATRP in the design of functional materials for biomedical applications. Prog. Polym. Sci. 2012;37(1):18-37. [DOI: 10.1016/j.progpoly msci.2011.08.001]

Vema G, Hassan PA. Self-assembled materials: design strategies and drug delivery perspectives. Phys. Chem. Chem. Phys. 2013;15:17016-28.

York AW, Zablocki KR, Lewis DR, Gu L, Uhrich KE, Prud'homme RK, Moghe PV. Kinetically assembled nanoparticles of bioactive macromolecules exhibit enhanced stability and cell-targeted biological efficacy. Adv. Mater. 2012;24(6):733-9. [DOI: 10.1002/adma.2011 03348]

Yu BG, Okano T, Kataoka K, Sardari S, Kwon GS. In vitro dissociation of antifungal efficacy and toxicity for amphotericin B-loaded poly(ethylene oxide)-blockpoly(b-benzyl-L-aspartate) micelles. J. Control. Release 1998;56(1-3):285-91. [DOI: 10.1016/s0168-3659(98)00 095-9] 